\title{
A Study on Solubility Enhancement Methods for Poorly Water Soluble Drugs
}

\author{
Praveen Kumar $^{1, *}$, Chhater Singh ${ }^{2}$ \\ ${ }^{1}$ S. D. College of Pharmacy \& Vocational Studies, Muzaffarnagar, India \\ ${ }^{2}$ Shri Venkateshwara University Rajabpur, Gajraula, India \\ *Corresponding author: praveensha77@gmail.com
}

Received January 14, 2013; Revised September 12, 2013; Accepted September 15, 2013

\begin{abstract}
It is generally recognized that poor solubility is one of the most frequently encountered difficulties in the field of pharmaceutics. Low solubility and subsequent unsatisfactory dissolution rate often compromise oral bioavailability. There are most therapeutic agents used to produce systemic effects by oral route that are the preferred way of administration owing to its several advantages and high patient compliance compared to other routes. However, poorly water-soluble drugs, when administered orally, have been shown to be slowly and unpredictably absorbed since their bioavailability is largely dependent on the dissolution process in gastrointestinal tract. This article demonstrates the various methods used to increase dissolution rates, preparation techniques of solid dispersion, and characterization methods of the solid dispersion.
\end{abstract}

Keywords: solubility, bioavailability, solid dispersion, dissolution, solvent evaporation method

Cite This Article: Praveen Kumar, and Chhater Singh, "A Study on Solubility Enhancement Methods for Poorly Water Soluble Drugs.” American Journal of Pharmacological Sciences 1, no. 4 (2013): 67-73. doi: 10.12691/ajps-1-4-5.

\section{Introduction}

The formulation of poorly water-soluble drugs has always been a challenging problem faced by pharmaceutical scientists and it is expected to increase because approximately $40 \%$ or more of the new chemical entities being generated through drug discovery programs are poorly water-soluble. The problem is even more intense for drug such as intraconazole and carbamazepine as they are poorly soluble in both aqueous and organic media, and for drugs having a $\log \mathrm{p}$ ( The logarithm of the ratio of the concentrations of the un-ionized solute in the solvents is called $\log \mathrm{P}$ ) value of 2 . Such drugs often have an erratic absorption profile and highly variable bioavailability because their performance is dissolution rate limited and is affected by the fed / fasted state of the patient [1,2].

When a drug is administered per-orally in solid dosage form such as tablet, capsules, or suspension it must be released from the dosage form and dissolved in the gastrointestinal fluids before it can be absorbed. The bioavailability of many poorly water-soluble drugs is limited by their dissolution rates, which are in turn controlled by surface area that they present for dissolution.

There are consecutive two processes can be identified to describe the oral absorption of drugs from solid dosage forms:

- Dissolution of the drug in vivo to produce a solution and

- Transport of the dissolved drug across the gastrointestinal membrane.
Each process can be characterized by a rate constant. If the rate of dissolution of the drug is significantly slower than the rate of absorption, the dissolution of the drug becomes the rate-limiting step in the absorption process [3]. Consequently, numerous attempts have been made to modify the dissolution characteristics of certain drugs in an effort to attain more rapid and more complete absorption4. And the particle size of the drug in of great importance in the transport from the gastrointestinal (GI) tract to the site of action by increasing the dissolution rate in the GI tract [3].

\section{Methods Used to Increase Dissolution Rate [5]}

\subsection{Complexation:}

A complex is a species of definite substrate-to-ligand stoichiometry that can be formed in an equilibrium process, in solution, and also in the solid state. Watersoluble ligands, such as cyclodextrins, can be used to solubilize water-insoluble substrate, such as ibuprofen.

\subsection{Cosolvents:}

A water-miscible organic solvent that is used to increase the aqueous solubility of a water-insoluble drug by utilizing the lipophilicity of organic solvent which is more affinity to the lipophilic water-insoluble drug.

\subsection{Emulsions:}


Colloidal dispersions of at least two immiscible phases, oil phase and aqueous phase, stabilized with the aid of a third component generally referred to as the emulsifying agent, or surfactant. Water-insoluble drugs with the oil soluble property can be solubilized in the oil phase.

\subsection{Microemulsions:}

Thermodynamically stable species, with much smaller droplet diameter (6 to $80 \mathrm{~nm}$ ) and thus are visually transparent or translucent. In addition to the aqueous phase, oily phase, and surfactant, they have a "cosurfactant," and the proportions of the components are chosen such that they are in a stable region of the respective phase diagram; oil content is generally 2-20\% percent. Unlike conventional emulsions, microemulsions form spontaneously on mixing with little or no mechanical energy applied.

\subsection{Micelles:}

A special form of self-aggregated complex in which the interactant is a surfactant, a molecule possessing both a nonpolar and a polar portion. A water-insoluble drug usually is affinity to nonpolar portion of the surfactant.

\subsection{Polymeric Micelles:}

The substance forms a polymeric micelle is usually copolymers rather than conventional surfactant. Polymeric micelle formation occurs as a result of two forces. One is an attractive force that leads to the association of molecules while the other one a repulsive force, prevents unlimited growth of the micelles to a distinct macroscopic phase. Amphilphilic copolymers form micellar structures through self-association of the insoluble segments when placed in a solvent such as water.

\subsection{Liposomes:}

Liposomes are vesicles formed by either synthetic or nature phospholipids. These phospholipid molecules arrange themselves spontaneously into bilayer structures in water, so that the hydrophobic tails are shielded from water by hydrophilic heads. Vesicles can consist of one or more phospholipid bilayers with hydrophilic and hydrophobic compartments. The hydrophobic compartments can carry or fill with hydrophobic or water-insoluble drugs while the hydrophilic compartments can load hydrophilic or water-soluble drugs.

\subsection{Pharmaceutical Salts:}

The salt is form by a water-insoluble drug, either a weak base or a weak acid, and a counterman, either a strong acid or a strong base. The resultant salt, as an ionized form in water, is usually more soluble than the non-ionized form.

\subsection{Pro-drugs:}

Prodrug is a drug with covalently bound, inactive moiety (the promoiety) which provides the desired pharmaceutical properties, where the promoiety must be removed upon administration to regenerate the parent drug in the body. The prodrug alternations possible following chemical modification of the parent drug include changes in the solubility properties.

\subsection{Particle Size Reduction:}

Particle size reduction offers a significant opportunity for formulators to solve the product development hurdles inherent with water-insoluble drugs. The drug absorption enhancement of the insoluble drug is achieved by increasing the specific surface area in order to increase the dissolution.

\subsection{Percolation:}

Percolation is a solubilizing system used in solid formulation to enhance drug dissolution by using a percolation matrix. By carefully identifying critical concentrations of solubilizing agents, which are related to percolation thresholds in a percolation system, the dissolution of the insoluble drug can be enhanced.

\subsection{Solid State Alternation:}

An insoluble drug usually possesses one or more polymorphs, solvents and one amorphous form. By identifying and selecting one of these forms, which has highest solubility, the solubility of the water-insoluble drug can be enhanced.

\subsection{Soft Gel Technology:}

Formulating the oily soluble drug in liquid formulation, which is filled in a soft gelatin capsule, can increase solubility of a water-insoluble drug, which is oil soluble.

\subsection{Solid Dispersion:}

A pharmaceutical solid dispersion is an intimate mixture of a drug substance (solute) with diluents, termed a carrier (solvent or continuous phase). Solid dispersion systems in which the drug is dispersed in solid watersoluble matrices either molecularly or as fine particles have also shown promising result in increasing bioavailability of poorly water-soluble drugs [5,6].

Among the techniques to increase aqueous solubility / dissolution rate the formulation of solid dispersions is one of the most popular ones. [7] When dissolving the solid dispersions, it is believed that the drug substance in released as small discrete units owing to a fast dissolution of the easily soluble carrier. If the drug solubility in the carrier is high enough, a so-called solid solution can be obtained. Such a preparation will then give a system similar to a molecular solution after the carrier has been dissolved. For such systems it has been claimed that the dissolution of the carrier is the rate - limiting step. The formation of solid solution is therefore normally restricted to relatively low concentration of drugs $[8,9]$.

\section{Solid Dispersion}

The term solid dispersion $[5,6]$ refers to the dispersion of one or more active ingredients in an inert carrier or matrix at solid state prepared by the melting (fusion), "solvent or the melting-solvent method." The dispersion of a drug or drugs in a solid diluents or diluents by 
traditional mechanical mixing is not included in this category. The solid dispersion may also be called solidstate dispersion.

\subsection{Advantages:}

The promising results of solid dispersion in solubility and dissolution rate enhancement of poorly soluble drugs can be attributed to various aspects:

- From amorphous structure replacing crystalline structure [10,11,12].

- To improved local solubility and wettability of the poorly soluble drug in the solid dispersion matrix $[13,14]$.

- From the ability of carrier functional groups to form interactions with the drug $[15,16]$.

- To the increase in glass transition temperature (Tg) $[17,18]$ of the solid dispersion mixture.

- Inhibited drug precipitation from supersaturated solution $[19,20]$ to resulting metastable drug polymorphous with higher solubility and dissolution rate in the presence of the carrier [21].

\subsection{Classification:}

Solid dispersions have been classified mainly into five major categories [22]:

- Simple eutectic mixtures,

- Solid solutions,

- Glass solutions of suspension,

- Compound or complex formations between the drug and the carrier,

- Amorphous precipitations of a drug in a crystalline carrier.

\subsubsection{Simple Eutectic Mixtures:}

A eutectic mixture of a sparingly water- soluble drug and a highly water- soluble carrier may be regarded thermodynamically as an intimately blended physical mixture of its two crystalline components. These components are assumed to crystallize simultaneously in very small particulate sizes. The increase in specific surface area, therefore, is mainly responsible for the increased rate of dissolution of a poorly water- soluble drug.

Differential thermal analysis (DTA) of binary mixtures normally exhibits two endotherms, but a binary mixture of eutectic composition usually exhibits a single major endotherm. In the case of a simple eutectic system, the thaw points of binary mixtures of varying compositions are equal to the eutectic temperature of the system [22].

\subsubsection{Solid Solutions:}

Solid solutions consist of a solid solute dissolved in a solid solvent. If the carrier is crystalline, a mixed crystal is formed because the two components crystallize together in a homogeneous one- phase system. Particle size is reduced in solid solution to molecular level, i.e., the dissolution of the drug occurs in the solid-state matrix. Hence, this system would be expected to yield much higher rates of dissolution than simple eutectic systems. In binary systems, where solid solution formation is evident, the phase diagram is characterized by the disappearance of thaw points at a temperature higher than the eutectic temperature. A marked increase in dissolution rates of the sparingly water-soluble drugs digitoxin, 17-methyl testosterone, hydrocortisone acetate, and predinisolone acetate when dispersed in PEG 6000. This is believed to be due to formation of colloidal or molecular dispersion of the drug in the carrier [23].

\subsubsection{Glass Solution:}

The principle of glass solution formation was first reported by Chiou and Riegelman to enhance drug dissolution and absorption. A glass solution is a homogeneous system in which a glassy or a vitreous form of the carrier solubilizes drug molecules in its matrix. PVP (Polyvinylpyrrolidone) dissolved in organic solvents undergoes a transition to a glassy state upon evaporation of the solvent $[24,25,26]$.

\subsubsection{Compound or Complex Formation:}

This system is characterized by complexation of two components in a binary system during solid dispersion preparation. The availability of a drug form the complex is dependent on the solubility, dissociation constant, and the intrinsic absorption rate the complex [26].

\subsubsection{Amorphous Precipitation:}

Amorphous precipitation occurs when the drug precipitates as an amorphous form in the inert carrier. The high-energy state of the drug in this system generally produces much greater dissolution rates than the corresponding crystalline forms of the drug.

The conversion of a drug to an amorphous form of coprecipitation resulting in increased dissolution has been reported for sulfisoxazole- PVP [27].

\section{Preparation of Methods of Solid Dispersion}

Generally, there are two methods employed to prepare the solid dispersion: fusion or solvent process.

\subsection{Fusion Process}

In the fusion method of preparation, the carrier is heated to a temperature just above its melting point and the drug is incorporated into the matrix. The mixture is cooled with constant stirring to homogeneously disperse the drug throughout the matrix. Several mechanisms could operate during the process of dispersion. If the drug has a high degree of solubility in the carrier, the drug could remain "dissolved" in the solid state, yielding what is known as a solid solution.

Particle size reduction under these conditions proceeds to the ultimate level leading to molecular dispersion of the drug in the carrier matrix. These systems show very high drug dissolution rates compared to control samples. If, on the other hand, the solubility of the drug in solid state is not so high, crystallites of the drug become dispersed in the matrix. Such systems show only moderate increases in dissolution rates.

A third mechanism is the conversion of a drug to an amorphous form in the presence of the matrix, again exhibiting different dissolution rates and solubility. Other factors that may play a role include solubilizing effect 
conferred by the carrier itself, improved wetting or decreased surface hydrophobicity, complexation, and crystallization of the drug in a metastable polymorphic form of altered thermodynamic properties.

An important limitation of the fusion method of preparation is the exposure of drugs to elevated temperatures, particularly if the carrier is a high-melting solid and the drug is heat-sensitive [3].

\subsubsection{Advantages:}

This method is very suitable for drugs and carrier that are miscible in the molten state, making melting of the ingredients very easy to accomplish. Preparing solid dispersion by the melt method is not time consuming. Hence many batches of the product can be prepared in a very short time. The method is also advantageous for compounds, which do not undergo significant thermal degradation.

\subsubsection{Disadvantages:}

The main disadvantages of the melt method include thermal degradation, sublimation, and polymeric transformation, which can affect the physicochemical properties of the drug including its rate of dissolution. The decomposition or thermal degradation is often composition dependent and affected by melting time and the rate of cooling. [8] In order to reduce decomposition to acceptable levels, melting may be carried out at a temperature just above the highest melting component of the dispersion, which completely melts both drug and the carrier.

The temperature at which the dispersion solidifies affects crystallization rates and may alter both the size of the crystals and the hardness of the dispersion. This may result in tacky or glassy and unmanageable dispersions, which will require storage at elevated temperature to facilitate hardening. Upon communition of such dispersions, crystallization may be induced resulting in modification of dissolution characteristics [3].

\subsection{Solvent Method:}

In the solvent method of preparation, the carrier and the active ingredient are dissolved in a suitable organic solvent. This solvent is evaporated at an elevated temperature or under vacuum. As the solvent is being removed, supersaturation occurs followed by simultaneous precipitation of the constituents resulting in a solid residue. The coprecipitate is then dried under vacuum to drive out any solvent freely adhering to the particle surface. However, there is a possibility of the formation of a solvate within the crystal lattice. This presents a problem in terms of pharmaceutical acceptance since most of the solvents used are non aqueous (organic) and toxic. Hence, removal of even trace amounts of the solvent is implied. Highly sensitive techniques such as differential scanning calorimetry (DSC), differential thermal analysis (DTA), thermogravimetric analysis (TGA), and less sensitive procedures like gravimetry and spectroscopy can be used to demonstrate complete solvent removal [28].

\subsubsection{Selection of Solvent:}

The choice of solvent and its removal rate are critical to the quality of dispersion. Since the chosen carriers are generally hydrophilic and the drugs are hydrophobic, the selection of a common solvent is difficult and its complete removal, necessitated by its toxic nature, is imperative. Certain solvents may plastisize polymeric carriers, e.g., Polyvinylpyrrolidone (PVP), making their complete removal even more difficult. Careful control of the temperature and rate of evaporation of solvents is essential in controlling the particle size of the drug, and although low temperature cannot always be avoided. The Tolbutamide- PVP dispersion showed an instability that varied to the evaporating temperature [3].

\subsubsection{Advantages:}

The procedure is suitable for drugs that are thermolabile; reduced pressure and lower temperatures can be used to evaporate solvent. For aqueous systems, frozen temperatures can be used to evaporate the solvent, which can enhance the integrity of the drug.

\subsubsection{Disadvantages:}

Finding a suitable solvent that will dissolve both the drug and the carrier is very difficult and sometimes impossible. This is because most of the carriers are hydrophilic whereas most of the drugs are hydrophobic organic substances. This may be further complicated by the fact that different polymorphic forms of the same drug may be obtained if different solvents are used. Spironolactone dispersions in polyvinylpyrollidone were evaporated from solutions of ethanol, acetonitrile, and chloroform, respectively. [29] The highest dissolution rate was provided by ethanolic dispersions, whereas the chloroform dispersion provided the lowest dissolution rate. After a suitable solvent has been found, the rate of its removal is very critical in some solid dispersion, and complete removal of the solvent is even more difficult to accomplish. Plasticization of some polymers such as polyvinylpyrrolidone has occurred with the use of some solvents. [30] This made removal of the solvent extremely difficult. The volume of organic solvent needed to dissolve a suitable amount of drug and carrier is very large in some cases, and the recovery of solvent may be economically prohibitive.

\subsection{Other Methods}

\subsubsection{Fusion - Solvent Method:}

In the fusion methods a carrier(s) is/are melted and the $\operatorname{drug}(\mathrm{s})$ is / are incorporated in the form of a solution. If the carrier is capable of holding a certain proportion of liquid yet maintaining its solid properties, and if the liquid is innocuous, the need for solvent removal is eliminated. Otherwise, this method faces the same criticism of solvent retention described before. This method is particularly useful for drugs that have high melting points or that are thermolabile. The feasibility of the method has been demonstrated for spironolactone and griseofulvin dispersions in polyethylene glycol 6000 [28].

\subsubsection{Spray Drying:}

In this type of preparation, the carrier and the active ingredient are dissolved or suspend in a suitable solvent. This solvent is evaporated by drying it to apply a stream of heated air to remove the solvent [31]. Due to the large 
surface area of the droplets, the solvent rapidly evaporates and solid dispersion is formed quickly.

\subsubsection{Lyophilization (Spray Freeze Drying Method):}

This method is used to avoid the heating during the preparation of thermosensitive drugs; spray freeze drying (SFD) has been successfully developed to prepare solid dispersions at ambient temperature, which was made significant development by the research work of William III (2-4). SFD technology involves the atomization of a feed liquid containing poorly water-soluble or insoluble APIs and excipients directly into a cryogenic liquid at ambient temperature to produce a frozen micronized powder that is subsequently dried. This process offers a variety of advantages compared to traditional technologies for solid dispersions, including amorphous structure and high surface area [32, 33, 34].

\subsubsection{Hot-melt Extrusion:}

It is a very common method used in the polymer industry. But Speiser [35,36] and Huttenrach [37] were the first persons who use this technology for pharmaceutical purpose.A melt extrusion consists of the following sections:

- An opening to feed raw materials,

- A heated barrel that consists of extruder screws to convey and mix the fed materials,

- And an exit port, which consists of an optional die to shape the extruding mass.

The Active ingredients and the carrier are fed into the heated barrel of extruder at a constant rate. When the mixture of active ingredient and the carrier is conveyed through heated screws, it is transformed into its "fluid like state". This state allows intimate and homogeneous mixing by the high shear of extruder screws. An exit port, which consists of an optional die, shapes the melt in the required form such as granules, pellets, films, or powder. An important advantage of the hot melt extrusion method is that the drug/carrier mix is only subjected to an elevated temperature for about one minute, which enables drug that are somewhat thermolabile to be processed.

\subsubsection{Electrostatic Spinning Method:}

Electrostatic spinning method is a straight-forward process for generating nanofibers. The popularity of this system is due to its ease of implementation, capability of being used with a variety of materials, convenience in obtaining composites of multiple components, and with secondary microstructures (such as core-sheath, side-byside, and island-in-sea). The applications of electrospun products are expanding, especially in areas relating to tissue engineering and drug delivery.38-42The fast-drying electrospinning process is able to "freeze" drug molecules randomly in the solid polymer fiber matrix into a state comparable with that in a liquid form. This is very useful for preventing phase separation, eg, recrystallization of either drug or matrix during removal of solvents [43-48].

\subsubsection{Supercritical Fluid Technology:}

Supercritical fluid technology (SCFT) is a new method to produce fine drug particles and is valuable for product quality. In the pharmaceutical field, the supercritical fluid technology was industrially applied in the early 1980’s.
In this technique the active ingredient and the carrier dissolve in a common solvent that is introduced into a particle formation vessel through a nozzle, simultaneously with $\mathrm{CO}_{2}$. When the solution is sprayed, the solvent is rapidly extracted by the supercritical fluid, resulting in the precipitation of solid dispersion particles on the walls and bottom of the vessel [49].

A supercritical fluid exists as a single phase above its critical temperature and pressure [50]. The most commonly used supercritical fluids include supercritical fluid carbon dioxide (SC-CO2), nitrous oxide, water, methanol, ethanol, ethane, propane, n-hexane and ammonia 18. SC-CO2 is a popular solvent or anti-solvent as it is safe, inexpensive, readily available, and an ideal substitute for many hazardous and toxic solvents. SC-CO2 exists when both the temperature and pressure equal or exceed the critical point of $31^{\circ} \mathrm{C}$ and 73 atm and has both gas-like and liquid-like qualities. It is this dual characteristic of supercritical fluids that provides the ideal conditions for extracting compounds with a high degree of recovery in a short period of time.

By controlling the level of pressure/temperature /modifier, SC-CO2 can dissolve a broad range of compounds, both polar and non-polar. At present, carbon dioxide technology is one of the fastest growing new process technologies being adopted by the pharmaceutical industry. Supercritical water is a unique medium for safe destruction of dangerous waste by total oxidation due to its special physicochemical properties. Various supercritical fluid technologies used in pharmaceutical processing include:

- Rapid expansion of supercritical solutions (RESS),

- Supercritical antisolvent (SAS) precipitation technique

- Particles from Gas Saturated Solutions (PGSS),

- Gas antisolvent system (SAS),

- Precipitation using compressed antisolvent (PCA),

- Aerosol solvent extraction system (ASES),

- Solution enhanced dispersion by supercritical fluids (SEDS),

- Supercritical antisolvent system with enhanced mass transfer (SAS-EM).

\subsubsection{Coating on Sugar Beads Using Fluidized Bed- Coating System:}

In this method fluidized bed-coating concept is involve. The drug and the active ingredient solution is sprayed onto the granular surface of excipients or sugar spheres to produce either granule ready for tableting or drug-coated pellets for encapsulation in one step. The method can be applied for both controlled- and immediate-release solid dispersions [51].

\subsection{Characterization of Solid Dispersions}

A number of methods have been used to characterize solid dispersions including.

\subsubsection{Differential Scanning Calorimetry:}

DSC is a frequently used thermoanalytical technique that generates data on melting endotherms and glass transitions. [52,53] Thermal analysis of samples can be carried out on a differential scanning calorimetry (DSC). 


\subsubsection{Powder X-ray Diffraction:}

Powder X-ray diffraction can be used to qualitatively detect material with long range order. Sharper diffraction peaks indicate more crystalline material. Recently developed X-ray equipment is semiquantitative.

\subsubsection{Microscopial Studies:}

Polarized light microscope has been used to examine the nucleation and crystal growth kinetics [54]. Polarized light microscope is more sensitive than DSC or XRD in detecting the onset of conversion from the amorphous to crystalline drug in solid solutions. Typically, samples can be examined under the polarized light microscope and images can be taken using digital camera and analyzed by the Motic Images Plus 2.0 software.

\subsubsection{Spectroscopic Methods, Especially I.R:}

FTIR spectra can be used to detect polymer-drug interactions by following the shift in vibrational or stretching bands of key functional groups. This method has been employed to identify polymer-drug interactions in solid molecular dispersions [55].

\subsubsection{Dissolution Rate Determination:}

Dissolution Calorimetry measures the energy of dissolution, which is dependent on the crystallinity of the sample. [56] Usually, dissolution of crystalline material is endothermic, whereas dissolution of amorphous material is exothermic.

\section{Conclusion}

There are many methods and procedures available to improve the solubility and enhance bioavailability. But Solid dispersion systems have been realized as extremely useful tool in improving the dissolution properties of poorly water-soluble drugs. In recent years, a great deal of knowledge has been accumulated about solid dispersion technology, but other methods like micronization, complexation, pro-drugs concepts also useful in pharmaceutical operations. The deep study is required to prevent the limitation of any method.

\section{References}

[1] Okonogi, S., Yonemochi, E., Oguchi, T., Puttipipatkhachorn, S., Yamamoto, K.,. Enhanced dissolution of ursodeoxycholic acid from the solid dispersion. Drug Dev. Ind. Pharm. 1997, 23(11), 1115-1121.

[2] Patravale, V.B., Date, A.A., Kulkarni, R.M.,. Nanosuspension: a promising drug delivery strategy. J. Pharm. Phamaco. 2004, 56(7), 827-840.

[3] Habib, M.J., Pharmaceutical solid dispersion Technology, Technomic Publishing Company, Inc. Lancaster, Pennsylvania (U.S.A.). 2001, pp. 1-36.

[4] Martin, M. T., Margarit, M.V., Salcedo, G.E., 2002. Characterization and solubility study of solid dispersions of flunarizine and plyvinyl pyrrolidone. Farmaco. , 2002, 57, 723727.

[5] Joshi, V.B., Tejwani, R.W., Davidovich, M., Saharabudhe, V.P., Jemal, M., Bathala, M.S., Varia, S.A., Serajuddin, A.T.M., Bioavalability enhancement of a poorly water-soluble drug by solid dispersion in polyethylene glycol-polysobate80 mixture. 2004, 269, 251-258.
[6] Chiou WL and Riegelman S. Pharmaceutical applications of solid dispersion systems. J. Pharm. Sci., 1971, 60(9): 1281-1302.

[7] Verheyan, S., Blaton, N., Kinget, R., and Mooter, G. V., Mechanism of increased dissolution of diazepam and tamezepam from polyethylene glycol 6000 solid dispersions. Int. J. Pharm. 2002, 266, 82-99.

[8] Dubois, J.L., and Ford, J.L., 1985. Similarities in the release rate of different drugs from polyethylene glycol 6000 solid dispersion. J.Phram. Pharmacol, 37, 494-496.

[9] Jung, J.Y., Yoo, S.D.F., Lee, S.H., Kin, K.H., Yoon, D.S., Lee, K.H. Enhanced solubility and dissolution rate of itraconazole by a solid dispersion technique. Int. J. Pharm. 1999, 187, 209-218.

[10] Leuner C, Dressman J, Improving drug solubility for oral delivery using solid dispersions, European Journal of Pharmaceutics and Biopharmaceutics, 2000, 50, 47-60

[11] $\mathrm{Yu} \mathrm{L,} \mathrm{Amorphous} \mathrm{pharmaceutical} \mathrm{solids:} \mathrm{preparation,}$ characterization and stabilization, Advanced Drug Delivery Reviews. 2001, 48, 27-42.

[12] Hancock BC, Zograf G, Characteristics and significance of the amorphous state in pharmaceutical systems, Journal of Pharmaceutical Sciences, 1997, 86, 1-12.

[13] Goldberg AH, Gibaldi M, Kanig JL, Mayersohn M, Increasing dissolution rates and gastrointestinal absorption of drugs via solid solutions and eutectic mixtures IV: chloramphenicol-urea system, Journal of Pharmaceutical Sciences, 1966, 55, 581-583.

[14] Verheyen S, Blaton N, Kinget R, Van den Mooter G, Mechanism of increased dissolution of diazepam and temazepam from polyethylene glycol 6000 solid dispersions, International Journal of Pharmaceutics. 2002, 249, 45-58.

[15] Konno H, Taylor LS, Influence of different polymers on the crystallization tendency of molecularly dispersed amorphous felodipine, Journal of Pharmaceutical Sciences. 2006, 95, 26922705.

[16] Tantishaiyakul V, Kaewnopparatv N, Ingkatawornwong S, Properties of solid dispersions of piroxicam in polyvinylpyrrolidone, International Journal of Pharmaceutics. 1999, 181, 143-151.

[17] Van den Mooter G, Wuyts M, Blaton N, Bussonc R, Grobetd P, Augustijnsa P, Kinget R, Physical stabilization of amorphous ketoconazole in solid dispersions with polyvinylpyrrolidone K25, European Journal of Pharmaceutical Sciences. 2001, 12, 261-269.

[18] Yoshioka M, Hancock BC, Zografi G, Inhibition of indomethacin crystallization in poly(vinylpyrrolidone) coprecipitates, Journal of Pharmaceutical Sciences. 1995, 84, 983-986.

[19] Simonelli AP, Mehta SC, Higuchi WI, Dissolution rates of high energy sulfathiazole-povidone coprecipitates II: characterization of form of drug controlling its dissolution rate via solubility studies, Journal of Pharmaceutical Sciences. 1976, 65, 355-361.

[20] Usui F, Maeda K, Kusai A, Ikeda M, Nishimura K, Yamamoto K, Inhibitory effects of water-soluble polymers on precipitation of RS-8359, International Journal of Pharmaceutics. 1997, 154, 5966.

[21] Mart'inez-Oha'rriz MC, Mart'in C, GonI MM, Rodr'iguezEspinosa C, Tros-Ilarduya MC, Zornoza A, Influence of polyethylene glycol 4000 on the polymorphic forms of diflunisal, European Journal of Pharmaceutical Sciences, 1999, 8, 127-132.

[22] Chiou, W.L., Riegelman, S., Pharmceutical applications of Solid dispersion systems. J. Pharm. Sci. 1971, 60, 1281-1302.

[23] Goldberg, A.H., Gibaldi, M., Kanig, J.L., Increasing dissolution rates and gastrointestinal absorption of drugs via solid solutions and eutectic mixtures. II. Experimental evalution of a eutectic mixture: urea-acetaminophen system. J. Pharm. Sci. 1966, 55, $482-487$.

[24] Chiou, W.L., Riegelman, S., Preparation and dissolution characteristics of several fast-release solid dispersions of griseofulvin. J. Pharm. Sci. 1969, 58(12), 1505-1510.

[25] Stupak, E.I., Bates, T.R.,. Enhanced absorption of digitoxin from orally administered digitoxin-polyvinylpyrrolidone coprecipitates. J. Pharm. Sci. 1973, 62(11), 1806-1809.

[26] Geneidi, A.S., Ali, A.A., Salama, R.B.,. Solid dispersions of nitrofurantoin, ethotoin, and coumarin with polyethylene glycol 6000 and their coprecipitates with povidone 25000. J. Pharm. Sci. 1978, 67(1), 114-116.

[27] Yamashia, K., Nakate, T., Okimoto, K., Ohike, A., Tokunaga, Y., Ibuki, R., Higaki, K., Kimura, T.,. Establishment of new preparation method for solid dispersion formulation of tacrolimus. Int. J. Pharm. 2003, 267, 79-91. 
[28] Higuchi, T., Ikeda, M. "Rapidly dissolving forms of digoxin: hydroquinone complex”. J. Pharm. Sci. 1974, 63(5), 809-811.

[29] Nelson, E., Knoechel, E.L., Hamlin, W.E., Wagner, J.G.,. Influence of the absorption rate of tolbutamide on the rate of decline of blood sugar levels in normal humans. Int. J. Pharm. 1962, 51, 509-514.

[30] Lin, S.L., Lachman, L., Swartz, C.J., Heubner, C.F.,. Preformulation investigation I. Rslation of salt forms and biological activity of an experimental antihypertensive. J. Pharm. Sci. 1972, 61(9), 1418-1422.

[31] T. Vasconcelos, B. Sarmento, and P. Costa, "Solid dispersions as strategy to improve oral bioavailability of poor water soluble drugs,” Drug Discov. Today, vol. 12 issues 23-24, 2007, pp. 10681075, doi:10.1016/j.drudis.2007, 09, 005.

[32] Rogers TL, Hu JH, Yu ZS, Johnston KP, Williams RO., III A novel particle engineering technology: spray-freezing into liquid. Int J Pharm. 2002, 242, 93-100.

[33] Rogers TL, Nelsen AC, Hu JH, Brown JN, Sarkari M, Young TJ, et al. A novel particle engineering technology to enhance dissolution of poorly water soluble drugs: spray-freezing into liquid. Eur J Pharm Biopharm. 2002, 54, 271-280

[34] Hu JH, Rogers TL, Brown J, Young T, Johnston KP, Williams RO. Improvement of dissolution rates of poorly water soluble APIs using novel spray freezing into liquid technology. Pharm Res. 2002, 19, 1278-1284.

[35] Speiser P. Galenische Aspecte der Arzneimittelwirkung. Pharm Acta Helv. 1966, 41, 321-342.

[36] Adel EI-Egakey M, Soliva M, Speiser P. Hot extruded dosage forms. Pharm Acta Helv. 1971, 46, 31-52.

[37] Huttenrauch R. Spritzgießverfahren zur Herstellung peroraler Retardpraperate. Pharmazie. 1974, 29, 297-302.

[38] Reference: 4. Seil JT, Webster TJ. Spray deposition of live cells throughout the electrospinning process produces nanofibrous three-dimensional tissue scaffolds. Int J Nanomedicine. 2011, 6, 1095-1099.

[39] Jannesari M, Varshosaz J, Morshed M, Zamani M. Composite poly(vinyl alcohol)/poly(vinyl acetate) electrospun nanofibrous mats as a novel wound dressing matrix for controlled release of drugs. Int J Nanomedicine. 2011, 6, 993-1003.

[40] Neves NM, Campos R, Pedro A, Cunha J, Macedo F, Reis RL. Patterning of polymer nanofiber meshes by electrospinning for biomedical applications. Int J Nanomedicine. 2007, 2, 433-448.

[41] Wang Y, Zhang C, Zhang Q, Li P. Composite electrospun nanomembranes of fish scale collagen peptides/chito-oligosaccharides: antibacterial properties and potential for wound dressing. Int $\mathrm{J}$ Nanomedicine. 2011, 6, 667-676.

[42] Teo WE, Ramakrishna S. A review on electrospinning design and nanofibre assemblies. Nanotechnology. 2006, 17, 89-106.

[43] Vasconcelos T, Sarmento B, Costa P. Solid dispersions as strategy to improve oral bioavailability of poor water soluble drugs. Drug Discov Today. 2007, 12, 1068-1075.
[44] Yu DG, Gao LD, White K, Brandford-White C, Lu WY, Zhu LM. Multicomponent amorphous nanofibers electrospun from hot aqueous solutions of a poorly soluble drug. Pharm Res. 2010, 27, 2466-2477.

[45] Yu DG, Yang JM, Branford-White C, Lu P, Zhang L, Zhu LM. Third generation solid dispersions of ferulic acid in electrospun composite nanofibers. Int J Pharm. 2010, 400, 158-164.

[46] Yu DG, Shen XX, Brandford-White C, White K, Zhu LM, Bligh SWA. Oral fast-dissolving drug delivery membranes prepared from electrospun polyvinylpyrrolidone ultrafine fibers. Nanotechnology. 2009, 20, 055104.

[47] Yu DG, Branford-White C, White K, Li XL, Zhu LM. Dissolution improvement of electrospun nanofiber-based solid dispersions for acetaminophen. AAPS Pharm Sci Tech. 2010, 11, 809-817.

[48] Yu DG, Branford-White C, Shen XX, Zhang XF, Zhu LM. Solid dispersions of ketoprofen in drug-loaded electrospun nanofibers. J Dispersion Sci Tech. 2010, 31, 902-908.

[49] T. Vasconcelos, B. Sarmento, and P. Costa, "Solid dispersions as strategy to improve oral bioavailability of poor water soluble drugs,” Drug Discov. Today. 2007, vol. 12 issues 23-24, pp. 10681075.

[50] F. V. T. Thakkar, T. G. Soni, M. C. Gohel, and T. R. Gandhi, "Supercritical fluid technology: A promising approach to enhance the drug solubility,” J. Pharm. Sci. Res., 2009, vol. 1, issue 4, pp. 1-14.

[51] H. Karanth, V. S. Shenoy, and R. R. Murthy, "Industrially feasible alternative approaches in the manufacture of solid dispersions: A technical report,” AAPS PharmSciTech., 2006, vol. 7 issue 4, Article 87, E31-38.

[52] Janssens S, de Armas HN, Remon JP, Van den Mooter G, The use of a new hydrophilic polymer, Kollicoat IR, in the formulation of solid dispersions of Itraconazole, European Journal of Pharmaceutical Sciences, 2007, 30, 288-294.

[53] Six K, Verreck G, Peeters J, Brewster M, Van Den Mooter G, Increased Physical Stability and Improved Dissolution Properties of Itraconazole, a Class II Drug, by Solid Dispersions that Combine Fast- and Slow-Dissolving Polymers, Journal of Pharmaceutical Sciences. 2004, 93, 124-131.

[54] Konno H, Taylor LS, Influence of different polymers on the crystallization tendency of molecularly dispersed amorphous felodipine, Journal of Pharmaceutical Sciences. 2006, 95, 26922705.

[55] Konno H, Taylor LS, Influence of different polymers on the crystallization tendency of molecularly dispersed amorphous felodipine, Journal of Pharmaceutical Science. 2006, 95, 26922705.

[56] Pikal MJ, Lukes AL, Lang JE and Gaines K Quantitative crystallinity determinations for betalactam antibiotics by solution calorimetry: correlations with stability. J. Pharm. Sci., 1978, 67(6): 767-73. 